\title{
A proper motion study of the globular cluster M 10
}

\author{
L. Chen ${ }^{1,2,3,4}$, M. Geffert ${ }^{4}$, J.J. Wang ${ }^{1,2,3}$, K. Reif ${ }^{4}$, and J.M. Braun ${ }^{4}$ \\ 1 Shanghai Astronomical Observatory, Chinese Academy of Sciences, Shanghai 200030, PR China \\ ${ }^{2}$ National Astronomical Observatories, Chinese Academy of Sciences, PR China \\ 3 Joint Lab for Optical Astronomy, Chinese Academy of Sciences, PR China \\ 4 Sternwarte der Universität Bonn, Auf dem Hügel 71, D-53121 Bonn, Germany
}

Received September 17, 1999; accepted May 4, 2000

\begin{abstract}
We present the first proper motion study of M 10 (NGC 6254). Absolute proper motions of about 532 stars in the field of the globular cluster M 10 were determined with respect to Hipparcos and ACT reference stars. In addition to photographic plates of Bonn and Shanghai also wide field CCD observations as second epoch plates were used. The wide field CCD observations show an accuracy comparable to that of the photographic plates. A good coincidence of the solutions based on reference stars from Hipparcos and from ACT was found. Our final proper motions allow a sufficient separation of cluster and field stars. Two population II Cepheids were confirmed to be members of M 10. The absolute proper motion of M 10 was determined and combined with its distance from the Sun and its radial velocity. The space motion and metallicity of M 10 indicates the characteristics of a halo object with an orbit reaching to a maximal $z$-distance of less than $3 \mathrm{kpc}$.
\end{abstract}

Key words: astrometry - globular clusters: individual: M 10 - Galaxy: kinematics and dynamics

\section{Introduction}

Globular clusters are important tracers of the chemical and dynamical evolution of the Milky Way. Since the orbits of globular clusters may have kept their characteristics from the early times of the formation of the Milky Way, we may extract from their kinematics some clues for the understanding of the origin of the Milky Way. However so far for only some 40 globular clusters absolute proper motions exist (Dinescu et al. 1999), which partly are of low quality (e.g. Dauphole et al. 1996). Nevertheless on the basis of a sample of 26 globular clusters Dauphole

Send offprint requests to: M. Geffert et al. (1996) found an indication of a metallicity gradient among the halo clusters and a mean rotation of about $+40 \mathrm{~km} \mathrm{~s}^{-1}$ for the complete globular cluster sample. Additional data especial for southern clusters were determined by Dinescu et al. (1999 and references herein).

The results of the Hipparcos mission offer new possibilities for the determination of absolute proper motions of globular clusters and other objects of interest for galactic kinematics. Since previous studies have used mainly extragalactic objects as proper motion zero points, the use of Hipparcos reference stars offers a unique possibility for the determination of absolute proper motions at low galactic latitutes without suitable extragalactic background objects. Geffert et al. (1997) have determined absolute proper motions of 10 globular clusters with respect to Hipparcos. These were combined with data of 5 other clusters and the kinematics of this sample was studied by Brosche et al. (1997) and Odenkirchen et al. (1997). A complete discussion of these data together with additional proper motions was given recently in Dinescu et al. (1999).

M 10 is a globular cluster located very near to the direction to the galactic center $\left(l=15^{\circ}, b=23^{\circ}\right)$, but at a distance of only $4.3 \mathrm{kpc}$ from the Sun. Due to its metallicity of $[\mathrm{Fe} / \mathrm{H}]=-1.52$ dex (Harris 1996) it belongs to the halo group of clusters according to Zinn's (1985) classification. The preliminary kinematical data of M 10 (Geffert et al. 1997) indicate a disk like motion, the most disk like motion found in the group of globular clusters, whose proper motions were determined using Hipparcos (Odenkirchen et al. 1997). However, this preliminary proper motion has the problem that only three to four Hipparcos stars could be used for the reduction of the plates. This made the previous solution very uncertain. Here we have included new photographic material (from Shanghai) and recent CCD observations to get a new determination of the absolute proper motion of M 10. Moreover, we use in addition to the Hipparcos catalogue the ACT catalogue (Urban et al. 1998). 
Table 1. Photographic material used in this work

\begin{tabular}{lrrr}
\hline Plate No. & Epoch & Emulsion & Telescope \\
\hline 149 & 1902.6 & & DR HL \\
406 & 1905.6 & & R SH \\
262 & 1916.5 & & DR HL \\
309 & 1917.5 & & DR HL \\
312 & 1917.5 & & DR HL \\
314 & 1917.5 & & DR HL \\
CL57016 & 1957.5 & 103a-O & R SH \\
CL57031 & 1957.6 & $103 a-O$ & R SH \\
CL57033 & 1957.6 & 103a-O & R SH \\
1232 & 1976.6 & IIa-O/BG 25 & DR HL \\
1874 & 1994.5 & IIa-O/BG 25 & DR HL \\
1875 & 1994.5 & IIa-O/BG 25 & DR HL \\
\hline
\end{tabular}

DR HL $=(D=0.3 \mathrm{~m}, f=5.1 \mathrm{~m})$ double refractor of Bonn. $\mathrm{R} \mathrm{SH}=(D=0.4 \mathrm{~m}, f=7.0 \mathrm{~m})$ refractor of Shanghai observatory.

The ACT catalogue, due to its denser coverage of the sky, allows the use of more reference stars for the determination of the absolute proper motions.

\section{Observations and the reduction}

Table 1 lists the photographic plates used in this work, while Table 2 indicates the observational data of the CCD frames. The plates were taken either with the $(D=0.3 \mathrm{~m}$, $f=5.1 \mathrm{~m}$ ) double refractor of Bonn, now located at Hoher List observatory, or with the $(D=0.4 \mathrm{~m}, f=7 \mathrm{~m})$ refractor of Shanghai. The CCD frames were taken with the WWFPP camera (Reif et al. 1994) at the $1.23 \mathrm{~m}$ telescope at Calar Alto and with a similar camera (HoLiCam) at Hoher List observatory. The use of the complete material allows a nearly uniform coverage of the epoch difference of 92 years. The limiting magnitude of the plates is of the order of $V=15.5 \mathrm{mag}$, while the corresponding one of the CCD frames ranges from $V=16$ to $19 \mathrm{mag}$. The refractor plates of Bonn were mainly scanned at the PDS 2020GM of University of Münster. The plates R1874 and R1875 with lower limiting magnitude have been measured at the ASCORECORD of Hoher List observatory. On these plates only 80 stars for each plate were measured. Stars were extracted and rectangular coordinates $x$ and $y$ were determined from the PDS measurements using standard procedures (e.g. Tucholke 1994). The first epoch plates of the Bonn refractor contained scratches and reseau lines, which led to problems for a significant number of the stars. Therefore for some of these stars no rectangular coordinates could be obtained. The plates from Shanghai were scanned at the PDS 1010MS of the Purple Mountain Observatory, Chinese Academy of sciences (see also Wang et al. 1999).

The determination of the rectangular coordinates $x$, $y$ of the stars on the CCD frames was performed for the observations from Hoher List by standard CCD reduction
Table 2. CCD observations used in this work. All CCD observations were made with the WWFPP camera (Reif et al. 1994)

\begin{tabular}{lclr}
\hline Telescope & Epoch & Filter & No. of frames \\
\hline 1 m Hoher List & 1996 & $V, B$ & 10 \\
1.23 m Calar Alto & 1994 & $R$ & 5 \\
\hline
\end{tabular}

techniques (DAOPHOT, IRAF) routines. Magnitudes, $x$ and $y$ positions were determined via PSF fit. The observations from Calar Alto were reduced earlier (Geffert et al. 1994) by the IMEX routine of the IRAF program package.

The astrometric reduction was performed by a central overlap algorithm. Due to the small field of the CCD frames we had to use only the plates in the first step of the reduction. A catalogue of positions and proper motions of 450 stars was established in this first step. In the following steps of the reduction the CCD frames were included. While for the plates only quadratic polynomials of the rectangular coordinates had to be taken into account, third order polynomials were necessary for the reduction of the CCD frames. The third order polynomials for the reduction of the CCD frames had to be used due to the distortion of the optics of the focal reducer of the WWFPP camera (Geffert et al. 1994). From the different position and time pairs we determined for each star for a certain epoch the mean position and the proper motion using least squares technique. All stars with proper motion errors larger than 4 mas/yr were omitted. The final catalogue contains 532 positions and proper motions of stars in the region of $\mathrm{M} 10$. The median of the internal errors was about $\pm 1 \mathrm{mas} / \mathrm{yr}$. From a plot of the proper motions versus magnitude no magnitude equation was found in our data.

We have performed two independent reductions of the M 10 data with reference stars from Hipparcos (ESA 1997) and the ACT catalogue (Urban et al. 1998). Although the Hipparcos stars proper motions are more accurate, they seemed to be of only limited usefulness for our work, since the majority of the plates contained only four Hipparcos stars, while about 17 stars could be used from the ACT catalogue. Therefore we consider both solutions as equivalent. Table 3 (the complete table is available in electronic form) gives the catalogue of our positions and proper motions for the complete field with respect to the ACT catalogue. The limiting magnitude of this catalogue is about $V=15.5 \mathrm{mag}$, which corresponds to the limiting magnitude of the first epoch plates. The size of the field is approximately $75 \times 75 \operatorname{arcmin}^{2}$ centered on M 10. We chose the ACT solution for the catalogue in Table 3 . The proper motions may be transfered to the Hipparcos system by adding $\Delta \mu_{\alpha} \cos \delta=-1.5 \mathrm{mas} / \mathrm{yr}$ and $\Delta \mu_{\delta}=+0.1 \mathrm{mas} / \mathrm{yr}$ to the proper motions from Table 3 . For the determination of the membership we will also use the solution based 
Table 3. The catalogue of positions and proper motions of 532 stars in the field of M 10. Four lines are given as an example. The complete catalogue is available in electronic form at VizieR (CDS; see Ochsenbein et al. 2000). The epoch of the positions is 1950

\begin{tabular}{|c|c|c|c|c|c|c|c|c|}
\hline \multirow[t]{2}{*}{ No. } & $\alpha_{2000}$ & $\delta_{2000}$ & \multirow{2}{*}{$\begin{array}{l}\sigma_{\alpha} \\
{[\mathrm{s}]}\end{array}$} & \multirow{2}{*}{$\begin{array}{l}\sigma_{\delta} \\
{\left[{ }^{\prime \prime}\right]}\end{array}$} & \multirow{2}{*}{$\begin{array}{c}\mu_{\alpha} \cos \delta \\
{[\mathrm{mas} / \mathrm{yr}]}\end{array}$} & \multirow{2}{*}{$\begin{array}{c}\mu_{\delta} \\
{[\mathrm{mas} / \mathrm{yr}]}\end{array}$} & \multirow{2}{*}{$\begin{array}{r}\sigma_{\mu_{\alpha} \cos \delta} \delta \\
{[\mathrm{mas} / \mathrm{yr}]}\end{array}$} & \multirow{2}{*}{$\begin{array}{c}\sigma \mu_{\delta} \\
{[\mathrm{mas} / \mathrm{yr}]}\end{array}$} \\
\hline & {$\left[\begin{array}{lll}\mathrm{h} & \mathrm{m} & \mathrm{s}\end{array}\right]$} & {$\left[\begin{array}{lll}0 & 1 & \prime \prime\end{array}\right]$} & & & & & & \\
\hline 1 & 165434.488 & -44520.57 & 0.004 & 0.04 & -33.2 & -27.5 & 1.7 & 1.2 \\
\hline 2 & 165446.910 & -35253.73 & 0.006 & 0.02 & 3.5 & 5.8 & 3.3 & 0.6 \\
\hline 3 & 165447.085 & -42306.77 & 0.002 & 0.01 & -5.5 & 4.4 & 1.1 & 0.3 \\
\hline 4 & 165448.326 & -41334.70 & 0.002 & 0.03 & -4.3 & -2.2 & 0.7 & 0.7 \\
\hline$:$ & $:$ & : & : & : & : & : & : & : \\
\hline
\end{tabular}

Table 4. Mean accuracy of the position of one star on a CCD frame determined from intercomparison of two CCD frames

\begin{tabular}{lrrrr}
\hline Frames & $\begin{array}{c}s_{\alpha} \\
{[\mathrm{mas}]}\end{array}$ & $\begin{array}{c}s_{\delta} \\
{[\mathrm{mas}]}\end{array}$ & $\begin{array}{c}\text { Lim. mag. } \\
(V)\end{array}$ & No. of stars \\
\hline $4236 / 4237$ & 62 & 64 & 16.5 & 1373 \\
$4238 / 4239$ & 120 & 120 & 18.5 & 3378 \\
$4240 / 4241$ & 63 & 67 & 16.2 & 1081 \\
$4242 / 4243$ & 100 & 80 & 17.0 & 1980 \\
\hline
\end{tabular}

on the ACT catalogue, while for the determination of the absolute proper motion of M 10 we will take the mean of both solutions.

\section{On the astrometric accuracy of the Hoher List CCD frames}

CCDs have been used in astrometry for several years, e.g. for the determination of parallaxes, double stars and for meridian circle observations (see references in Geffert 1998). However most of these observations are based on CCD observations with fields of $\left(10^{\prime}\right)^{2}$ and smaller. Since our study uses CCD observations of fields with a size at least $20^{\prime} \times 20^{\prime}$ it seems necessary to evaluate the accuracy, which may be obtained with such telescope detector combinations. While the CCD frames of Calar Alto were already tested in an earlier study (Geffert et al. 1994), we will concentrate here on the Hoher List observations.

In a first step we have compared positions of stars from pairs of CCD frames. The positions of one frame were transformed by an affine transformation to a second frame and the rms of the differences were calculated for each coordinate. Under the assumption that both frames contribute with equal weight to the differences, we have calculated from the rms the mean uncertainty of one position on one frame. These are given in Table 4 for several CCD pairs with nearly identical limiting magnitudes. In this comparison we included all stars which were detected on the CCD frames.

In a second test we consider only the stars which contribute to the final catalogue of our investigation. These
Table 5. Mean deviation $\left(\Delta_{\alpha}, \Delta_{\delta}\right)$ and rms $\left(\sigma_{\alpha}, \sigma_{\delta}\right)$ of the positions of different CCD frames in the last step of the iteration. The data are based on the mean of about 270 stars

\begin{tabular}{lrrrrr}
\hline CCD frame & $\begin{array}{c}\Delta_{\alpha} \\
{[\mathrm{mas}]}\end{array}$ & $\begin{array}{c}\sigma_{\alpha} \\
{[\mathrm{mas}]}\end{array}$ & $\begin{array}{c}\Delta_{\delta} \\
{[\mathrm{mas}]}\end{array}$ & $\begin{array}{c}\sigma_{\delta} \\
{[\mathrm{mas}]}\end{array}$ & Colour \\
\hline 4236 & +10 & 70 & -7 & 90 & $B$ \\
4237 & +6 & 70 & -5 & 70 & $B$ \\
4238 & +7 & 80 & 0 & 90 & $B$ \\
4239 & +6 & 100 & +2 & 50 & $B$ \\
4240 & +7 & 80 & 0 & 50 & $V$ \\
4241 & -2 & 40 & -1 & 50 & $V$ \\
4242 & -0 & 90 & +5 & 50 & $V$ \\
4243 & +3 & 40 & +6 & 90 & $V$ \\
\hline
\end{tabular}

are stars at the brighter end of the magnitude distribution. Reducing the plates with one catalogue (ACT or Hipparcos) leads to positions of the stars of each plate/CCD frame in a common system. The position and proper motion of each star in our final solution described in Sect. 2 are determined by a fit to the positions and epochs of the different plates/CCD frames for each star. The mean position and proper motion of each star is then used to update the positions of each star for the epoch of the individual plates/CCD frames. For each plate the mean and rms of the positional differences from the initial positions are determined. The rms will give an indication of the accuracy of each individual plate/CCD frame. Table 5 summarizes for the Hoher List frames the mean and standard deviations for each frame.

Table 5 shows a slight difference in the $B$ and $V$ frames of the order of a few mas. Nevertheless the systematic deviations between the $B$ and $V$ frames are small. The positional accuracy of each star is of the order of one tenth of a pixel. This value is a little bit larger with respect to other studies (e.g. Geffert 1998). The reason may be the crowding in the region of M 10. In general, the accuracy of one single frame is of the order of the accuracy of one refractor plate, which justfies the use of CCD frames for the second epoch observation. 


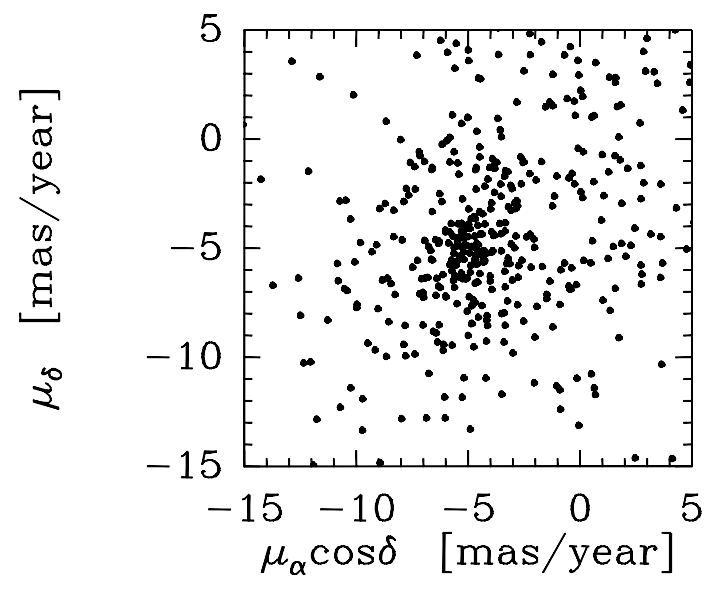

Fig. 1. The vector-point-plot diagram of all stars in the field of M 10

\section{Separation of cluster and field stars}

Figure 1 gives the vector-point-plot diagram of all our proper motions indicating the concentration of the cluster stars. For the separation of cluster and field stars we have concentrated our investigation on stars up to the limiting radius of $14^{\prime}$ (Webbink 1988) around the cluster centre. 284 stars remained in the data sample. For these stars a bivariate Gaussian fitting to the proper motions was performed using the method of Sanders (1971). Table 6 gives the result of the analysis. The cluster standard deviations are larger than the internal errors of the proper motion. This difference is clearly not an indication of a possible detection of internal motions of stars in the cluster. More likely these differences are caused by crowding effects and undetected systematic errors of the different telescopes used in this investigation.

M 10 is a globular cluster with three slow variable stars (Clement et al. 1985) and several UV bright stars (Zinn et al. 1972; Harris et al. 1983). Our sample contains only the variable stars $V 2$ and $V 3$ (see references in Clement et al. 1985). The other special stars are located in crowded regions, below the limiting magnitude, or exhibit too large proper motion errors. Both variable stars $V 2, V 3$ have according to our investigation a membership probability of 97\%. This confirms the earlier suggestion (Clement et al. 1985) that these stars are of W-Virginis type, although $V 3$ has a very unusual period of 7.8 days.

\section{The space motion of $M \mathbf{1 0}$}

The first absolute proper motion of M 10 based on the Hipparcos system was given in Geffert et al. (1997), which was later used in Odenkirchen et al. (1997) and Dinescu et al. (1999). However, as mentioned in Odenkirchen et al.
Table 6. Parameters of the fit to the bivariate Gaussian distribution of the proper motions of stars with $R<14^{\prime}$ around $\mathrm{M} 10 . n_{\mathrm{c}}$ and $n_{\mathrm{f}}$ denote the number of the cluster and field stars, $x_{\mathrm{c}}, y_{\mathrm{c}}$ the mean cluster proper motion in $\alpha$ and $\delta$, respectively, $x_{\mathrm{f}}, y_{\mathrm{f}}$ the mean proper motion of field stars, $\sigma_{x \mathrm{c}}$, $\sigma_{y \mathrm{c}}$ the Gaussian widths of the cluster distribution, and $\sigma_{x \mathrm{f}}$, $\sigma_{y \mathrm{f}}$ the widths of the field star distribution. The $x$-direction is the direction of right ascension, the $y$ direction is the declination axis. The units are mas/yr

\begin{tabular}{lrlr}
\hline Parameter & Value & Parameter & Value \\
\hline$n_{\mathrm{c}}$ & 193 & $n_{\mathrm{f}}$ & 88 \\
$x_{\mathrm{c}}$ & -4.9 & $x_{\mathrm{f}}$ & -3.5 \\
$y_{\mathrm{c}}$ & -5.3 & $y_{\mathrm{f}}$ & -3.3 \\
$\sigma_{x \mathrm{c}}$ & 1.8 & $\sigma_{x \mathrm{f}}$ & 7.0 \\
$\sigma_{y \mathrm{c}}$ & 2.0 & $\sigma_{y \mathrm{f}}$ & 7.1 \\
\hline
\end{tabular}

(1997), the result of M 10 was only preliminary due to the small number of Hipparcos stars used for the reduction of the plates. The use of additional plates from Shanghai has led to a significant lower proper motion in declination. However, even for this sample of plates the solution is only moderately stable: omitting one reference star leads to an absolute proper motion of M 10, which differs from the original solution by about more than the mean uncertainties of the absolute proper motions. Fortunately, this is not the case for the denser ACT catalogue. In summary, the use of the ACT catalogue resulted in a more stable solution, while the use of Hipparcos stars provided the more direct link to an extragalactic reference frame. The catalogues based on the ACT catalogue (Urban et al. 1998) and on the Hipparcos catalogue (ESA 1997) contributed therefore with equal weight to the final absolute proper motion of the globular cluster M 10. We obtained a mean absolute proper motion of $\mu_{\alpha} \cos \delta=-5.5 \pm 1.2 \mathrm{mas} / \mathrm{yr}$ and $\mu_{\delta}=-6.2 \pm 1.2 \mathrm{mas} / \mathrm{yr}$. Together with the distance from the Sun of $4.3 \mathrm{kpc}$ and a radial velocity of $+76 \mathrm{~km} \mathrm{~s}^{-1}$ (Harris 1996) we have calculated the velocity components in a system of galactic standard at rest $(U, V, W)$, peri- and apogalactic distances $R_{\mathrm{p}}, R_{\mathrm{a}}$, eccentricity $e$ and the $z$-component of the angular momentum of the orbit using a simple logarithmic galactic mass model (see e.g. Dauphole et al. 1996). These data are shown in Table 7. Figure 2 gives the orbit of M 10 integrated over 10 Gyr backwards according to the method of Allen \& Santillán (1991) using the programme of Odenkirchen et al. (1997).

\section{Discussion}

Our results demonstrate the importance of the new astrometric catalogues (Hipparcos, ACT) for the determination of absolute proper motions of objects located in the galactic plane. For these objects no extragalactic link of the proper motion system is available due to the absence 
Table 7. Velocity components in a system of galactic standard at rest and kinematical data of the orbit of globular cluster M 10 . A simple logarithmic galactic mass model was used (see e.g. Dauphole et al. 1996). (Note that $U$ points from Sun to galactic centre! A positive $z$ component of the angular momentum indicates a prograde rotation in the Galaxy)

\begin{tabular}{cccccccr}
\hline Object & $\begin{array}{c}U \\
{\left[\mathrm{~km} \mathrm{~s}^{-1}\right]}\end{array}$ & $\begin{array}{c}V \\
{\left[\mathrm{~km} \mathrm{~s}^{-1}\right]}\end{array}$ & $\begin{array}{c}W \\
{\left[\mathrm{~km} \mathrm{~s}^{-1}\right]}\end{array}$ & $\begin{array}{c}R_{\mathrm{a}} \\
{[\mathrm{kpc}]}\end{array}$ & $\begin{array}{c}R_{\mathrm{p}} \\
{[\mathrm{kpc}]}\end{array}$ & $e$ & $\begin{array}{c}I_{z} \\
{\left[\mathrm{kpc} \mathrm{km} \mathrm{s}^{-1}\right]}\end{array}$ \\
\hline $\mathrm{M} 10$ & $+111 \pm 10$ & $+90 \pm 18$ & $+63 \pm 18$ & $5.4 \pm 0.2$ & $2.3 \pm 0.4$ & $0.41 \pm 0.08$ & $+533 \pm 70$ \\
\hline
\end{tabular}

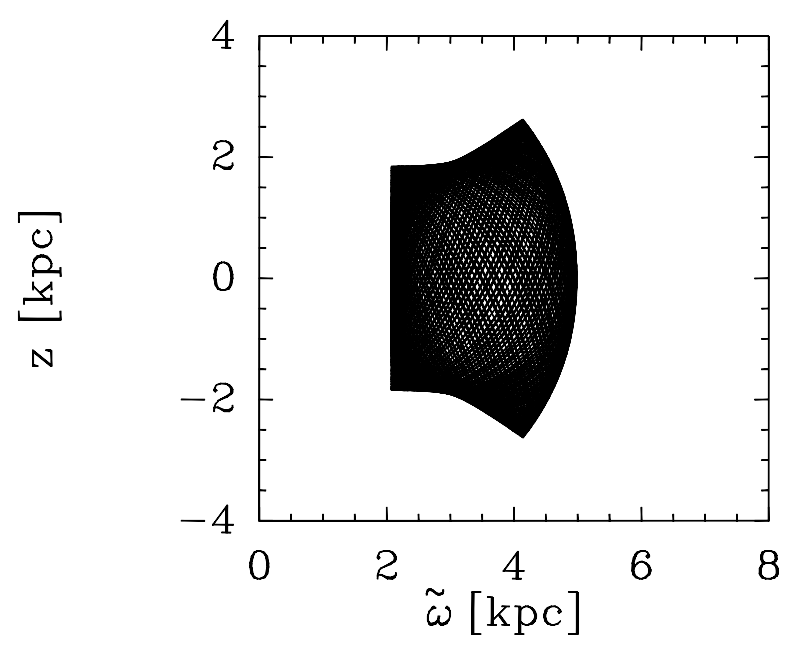

Fig. 2. The meridional section of the orbit of M 10 calculated backwards over $10 \mathrm{Gyr}$

of suitable objects. We show that the differences between the solutions based on the ACT catalogue and the corresponding one based on the Hipparcos catalogue are small. Although this result has to be checked using data of other fields, it would offer a range of applications for the ACT catalogue because of its larger number of stars. Moreover, even reaching only moderate accuracy on the wide field CCD frames with respect to small field CCD observations, our results indicate that these observations may supersede the photographic plates in near future.

The new data of M 10 show an eccentricity of the orbit of 0.41 , which would place the cluster now in the range of eccentricities from 0.4 to 0.8 found for the majority of the globular clusters (Odenkirchen et al. 1997). The rotational velocity changed to $\Theta=+111 \pm 17 \mathrm{~km} \mathrm{~s}^{-1}$ with respect to the $+144 \mathrm{~km} \mathrm{~s}^{-1}$ found in Odenkirchen et al. (1997). According to its moderate eccentricity and rotational velocity, M 10 belongs rather to the halo class of objects. This is in line with its metallicity. However, the $z$-distance from the galactic plane does not exceed $3 \mathrm{kpc}$, which would be more characteristic for a thick disk object.

As noted already in Dinescu et al. (1999) recent age determinations (Hurley et al. 1989; Richer et al. 1996; Chaboyer et al. 1996; Buonano et al. 1998) agree that M 10 is an old halo cluster. Together with NGC 6626 ,
NGC 6752 (Dinescu et al. 1999) and M 71, which has according to Geffert \& Maintz (2000) a much higher age, M 10 establishes a group of high age globular clusters with orbits of a disk or thick disk character. This result would imply that at the time when the oldest globular clusters were born, the Milky Way had already a gas distribution with a disk component, where the gas enabled the formation of clusters. On the other hand the recent age determination by Rosenberg et al. (1999) places M 10 in the middle of the age distribution of globular clusters.

Piotto \& Zoccali (1999) have found an unusual steep luminosity function of M 10 in comparison with other clusters. Our data indicate that this luminosity function was generated more likely by internal dynamics of the cluster than by the motion in the Galaxy. If the luminosity function would be affected by the crossing of the cluster through the galactic plane, one would rather expect a more flat luminosity function for M 10 due to the evaporation of faint stars as it was found recently for the globular cluster NGC 6712 (De Marchi et al. 1999).

Acknowledgements. We are indebted to H.-J. Tucholke (Bonn) for measuring some of the refractor plates. It is a pleasure to thank K.S. de Boer (Bonn) for helpful discussions. This research has made use of the Simbad database (see Wenger et al. 2000), operated at CDS, Strasbourg, France.

\section{References}

Allen C., Santillián, 1991, RMxA 22, 255

Brosche P., Odenkirchen M., Geffert M., Tucholke H.-J., 1997, Space motions and orbits of galactic globular clusters in: Hipparcos Venice 97, ESA SP-402. ESA Publications Division, Noordwijk, p. 531

Buonanno R., Corsi C.E., Pulone L., Fusi Pecci F., Bellazini M., 1998, A\&A 333, 505

Chaboyer B., Demarque P., Sarajedini A., 1996, ApJ 459, 559

Clement C.M., Sawyer Hogg H., Wells T.R., 1985, AJ 90, 1238

De Marchi G., Leibundgut B., Paresce F., Pulone L., 1999, A\&A 343, L9

Dauphole B., Geffert M., Colin J., Ducourant C., Odenkirchen M., Tucholke H.-J., 1996, A\&A 313, 119

Dinescu D.I., Girard T.G., van Altena W.F., 1999, AJ 117, 1792

ESA, 1997, The Tycho and Hipparcos Catalogue, ESA SP1200

Geffert M., 1998, CCD astrometry, in: The Message of the Angles - astrometry from 1798 to 1998 , Proc. of the spring 
meeting of the Astronomische Gesellschaft, Brosche P. et al. (eds). Verlag Harri Deutsch, Thun und Frankfurt am Main, p. 181

Geffert M., Reif K., Domgörgen H., Braun J.M., 1994, AG Abstr. Ser. 10, 125

Geffert M., Hiesgen M., Colin J., Dauphole B., Ducourant C., 1997, Absolute proper and space motions of globular clusters, in: ESA SP-402. ESA Publications Division, Norrdwijk, p. 579

Geffert M., Maintz G., 2000, A\&AS 144, 227

Harris W.E., 1996, AJ 112, 1487

Harris H.C., Nemec J.M., Hesser J.E., 1983, PASP 95, 256

Hurley D.J.C., Richer H.B., Fahlmann G.G., 1989, AJ 98, 2124

Ochsenbein F., Bauer P., Marcout J., 2000, A\&AS 143, 23

Odenkirchen M., Brosche P., Geffert M., Tucholke H.-J., 1997, New Astron. 2, 477

Piotto G., Zoccali M., 1999, A\&A 345, 485

Reif K., Müller P., Leiner T., Herkendell W., Bagschik K., 1994, AG Abstr. Ser. 10, 243
Richer H.B., et al., 1996, ApJ 463, 602

Rosenberg A., Saviane I., Piotto G., Aparicio A., 1999, AJ 118, 2306

Sanders W., 1971, A\&A 14, 226

Tucholke H.-J., Brosche P., Scholz R.-D., 1994, A\&AS 104, 161

Urban S.E., Corbin T.E., Wycoff G.L., 1998, AJ 115, 2161

Wang J.J., Chen L., Wu Z.Y., Gupta A.C., Geffert M., 1999, A\&AS 142,373

Webbink R.F., 1988, Kinematics of the Galactic Globular Cluster System. in: Proc. IAU Symp. 126, The HarlowShapley Symposium on Globular Cluster Systems in Galaxies, Grindlay J.E., Davis Philip A.G. (eds.), IAU Symp. 126. Reidel, Dordrecht, p. 49

Wenger M., Ochsenbein F., Egret D., et al., 2000, A\&AS 143, 9

Zinn R., 1985, ApJ 293, 424

Zinn R.J., Newell E.B., Gibson J.B., 1972, A\&A 18, 390 